\title{
C9orf72 expansion differentially affects males with spinal onset amyotrophic lateral sclerosis
}

\author{
James Rooney, ${ }^{1}$ Isabella Fogh, ${ }^{2}$ Henk-Jan Westeneng, ${ }^{3}$ Alice Vajda, \\ Russell McLaughlin, ${ }^{1}$ Mark Heverin, ${ }^{1}$ Ashley Jones, ${ }^{2}$ Ruben van Eijk, ${ }^{3}$ Andrea Calvo, ${ }^{4}$ \\ Letizia Mazzini, ${ }^{5}$ Christopher Shaw, ${ }^{2}$ Karen Morrison, ${ }^{6}$ Pamela J Shaw, ${ }^{7}$ \\ Wim Robberecht, ${ }^{8,9}$ Phillip Van Damme, ${ }^{8,10}$ Ammar Al-Chalabi, ${ }^{2}$ \\ Leonard van den Berg, ${ }^{3}$ Adriano Chiò, ${ }^{4}$ Jan Veldink, ${ }^{3}$ Orla Hardiman ${ }^{1,11}$
}

\begin{abstract}
- Additional material is published online only. To view please visit the journal online (http://dx.doi.org/10.1136/ jnnp-2016-314093)
\end{abstract}

For numbered affiliations see end of article.

Correspondence to Dr James Rooney, Academic Unit of Neurology, Trinity Biomedical Sciences Institute, Trinity College, 152-160 Pearse Street, Dublin 2, Dublin, D02 R590 Ireland; jrooney@rcsi.ie

Received 26 May 2016 Revised 19 August 2016 Accepted 30 August 2016 Published Online First 23 September 2016

\section{SLinked}

http://dx.doi.org/10.1136/ jnnp-2016-314685

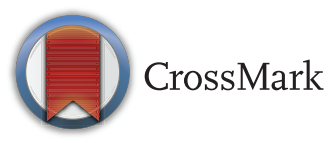

To cite: Rooney J, Fogh I, Westeneng $\mathrm{H}-\mathrm{J}$, et al.

J Neurol Neurosurg Psychiatry 2017:88:295-300.

\section{ABSTRACT}

Introduction The C9orf72 repeat expansion has been reported as a negative prognostic factor in amyotrophic lateral sclerosis (ALS). We have examined the prognostic impact of the C9orf72 repeat expansion in European subgroups based on gender and site of onset.

Methods C9orf72 status and demographic/clinical data from 4925 patients with ALS drawn from 3 prospective ALS registers (Ireland, Italy and the Netherlands), and clinical data sets in the UK and Belgium. Flexible parametric survival models were built including known prognostic factors (age, diagnostic delay and site of onset), gender and the presence of an expanded repeat in C9orf72. These were used to explore the effects of C9orf72 on survival by gender and site of onset. Individual patient data (IPD) meta-analysis was used to estimate HRs for results of particular importance.

Results 457 (8.95\%) of 4925 ALS cases carried the C9orf72 repeat expansion. A meta-analysis of C9orf72 estimated a survival HR of 1.36 (1.18 to 1.57) for those carrying the expansion. Models evaluating interaction between gender and C9orf72 repeat expansions demonstrated that the reduced survival due to C9orf72 expansion was being driven by spinal onset males (HR 1.56 (95\% Cl 1.25 to 1.96$)$.

Conclusions This study represents the largest combined analysis of the prognostic characteristics of the C9orf72 expansion. We have shown for the first time that the negative prognostic implication of this variant is driven by males with spinal onset disease, indicating a hitherto unrecognised gender-mediated effect of the variant that requires further exploration.

\section{INTRODUCTION}

Amyotrophic lateral sclerosis (ALS) is a debilitating disease with a poor prognosis. Progress towards developing new treatments has been limited both by disease heterogeneity, and by the likely interaction between genetic and environmental factors in disease pathogenesis. ${ }^{1}$ A pathological expansion of a hexanucleotide repeat in the C9orf 72 gene $^{2}{ }^{3}$ accounts for up to $10 \%$ of those with ALS in populations of European extraction, and is associated with a distinctive clinical phenotype that includes frontotemporal dementia (FTD) in some instances. ${ }^{4-10}$ Although the C9orf72 repeat expansion has been shown to be an important negative prognostic factor in survival analyses, ${ }^{4-10}$ until now no study has been sufficiently large to permit robust analysis of interactions between the variant and demographic features including age, gender and site of onset. Here, we used our combined clinical data sets to determine whether the presence of the expanded variant differentially modulates survival based on gender and site of onset.

\section{METHODS}

Data sources/case capture

Clinical data from ALS cases incident from January 2000 to April 2015 were collected from Belgium, Ireland, Italy, Netherlands and the UK. All patients fulfilled the diagnostic criteria for ALS, and core data elements as defined by the European Network for the Cure of ALS (ENCALS consortium) were harmonised across data sets for consistency based on existing consortia agreements. ${ }^{11}$ Owing to the lack of an agreed international definition of 'familial ALS', and given that a previous populationbased familial aggregation analysis from Ireland demonstrated a much higher familial ALS occurrence $(16 \%)$ than usually recognised, ${ }^{12}$ we did not exclude cases based on family history. The Belgian and UK cases were collected from clinical research centre cohorts, while the Dutch, Irish and Italian cases were sourced from the prospective population-based national registers. ${ }^{13-19}$

In accordance with existing Consortia agreements, data were collated using the following variables: age of onset, date of onset, date of diagnosis, date of death/last known follow-up date, site of onset, revised El-Escorial diagnostic category (except Belgium) and C9orf72 status (normal or expanded). For all study participants, C9orf72 status was determined by repeat primed PCR as described previously (with individual laboratory-based validation and quality control by Southern blot analyses). ${ }^{3}$

\section{Survival analysis strategy}

Initially exploratory models were constructed using Cox proportional hazards regression to explore the effect of different time of entry to the studies. Cox models were generated including known important survival covariates including age of onset, site of onset, diagnostic delay and C9orf72. Cox models were compared using a likelihood ratio test, and by testing the validity of the proportional hazards assumption of each covariate at each timescale. 
A base model using Royston-Parmar flexible parametric regression ${ }^{20}$ was built on the preferred timescale, with a proportional hazards scale and a number of degrees of freedom selected by comparison of the Akaike information criterion and Bayesian information criterion from models with increasing degrees of freedom, and the variance-covariance matrix clustered by country. Survival follow-up was limited to 5 years from entry. Models were then built to explore the effect of C9orf 72 status in sex and site of onset subgroups. The stpm $2^{21}$ and $i p d-$ metan $^{22}$ commands from Stata MP V.14.0 were used to perform the survival analysis and produce the meta-analysis graphs, while the ggplot $2^{23}$ package in R V.3.1.1 was used to generate selected final graphs.

\section{RESULTS}

\section{Descriptive statistics and basic survival model}

In total, 5106 ALS cases met the inclusion criteria, of which 457 (8.95\%) carried the C9orf72 repeat expansion. Breakdown of the demographics of the overall cohort by country is shown in table 1 . Missing values were minimal affecting 181 cases (3.5\%). Online supplementary information 1 summarises the basic survival model. An individual patient data (IPD) meta-analysis of C9orf72 status in the base model estimated an HR of 1.36 (1.18 to 1.57 ) for those carrying the expansion versus those not.

\section{C9orf72, gender and site of onset subgroup analysis}

Survival curves were generated to evaluate the effect of C9orf 72 status on gender and site of onset (figure 1) which suggested a three-way interaction. Therefore, gender, site of onset and C9orf72 status were categorised into one variable with eight levels as demonstrated in Online supplementary information 2 table 1. Through comparison of survival curves, redundant subgroups were combined (see online supplementary information 2), leaving three groups: spinal onset males with the C9orf 72 expansion, other spinal onset patients and all bulbar onset patients. Survival curves for these groups showed that male spinal onset patients with the C9orf72 repeat expansion had a prognosis distinct from other spinal onset patients and similar to bulbar onset patients (figure 2). Meta-analysis calculated a survival HR of 1.56 (95\% CI 1.25 to 1.96) for male spinal onset patients with the C9orf72 repeat expansion (figure 3). The finding was in the same direction in each country, although only the pooled estimate was statistically significant (figure 3 ).

The median ages and distribution of diagnostic delay across the final subgroups is shown in table 2. While age of onset was oldest in the bulbar onset group and youngest in the male spinal onset C9orf72 expanded group, the male spinal onset C9orf72 expanded group also had the highest proportion in the 'short' diagnostic delay category, consistent with the finding that the C9orf72 expansion differentially affects disease course in a genderspecific manner. Adjustment for the El-Escorial category (table 3) did not substantially alter the HR (1.57 CI 1.26 to 1.97$)$.

\section{DISCUSSION}

Previously, studies have shown that people with ALS carrying a C9orf72 repeat expansion in blood present at a younger age and have reduced survival when compared to patients without the expanded variant (table 4). However, studies until now have not been sufficiently powered to determine whether the expanded variant differentially affects outcome in subgroups based on gender and site of onset. Our findings demonstrate an intriguing and previously unrecognised interaction between the expanded variant and male patients with spinal onset disease, which appears to drive the overall survival effect. Within this cohort, the median age of onset was 59.3 and the median survival was 2.29 years. This compared to a median age of onset of 62.3 and median survival of 2.77 years in all other spinal onset disease, and a median age of onset of 65 and median survival of 2.38 years in all bulbar onset disease. Moreover, and contrary to the usual pattern in young onset disease, male spinal onset C9orf72 expanded cases were also more likely to have experienced a shorter diagnostic delay, suggesting rapidly progressing disease.

Female gender has previously been reported as an independent predictor of faster functional decline in ALS $;{ }^{24}$ however, our observation of an interaction between site of onset, gender and C9orf72 has not been previously noted, possibly due to

Table 1 Baseline demographics of C9orf72-tested ALS cases by country

\begin{tabular}{|c|c|c|c|c|c|c|c|}
\hline Variable & $\begin{array}{l}\text { Belgium } \\
\mathrm{n}=482\end{array}$ & $\begin{array}{l}\text { Ireland } \\
\mathrm{n}=645\end{array}$ & $\begin{array}{l}\text { Italy } \\
\mathrm{n}=897\end{array}$ & $\begin{array}{l}\text { The Netherlands } \\
n=2153\end{array}$ & $\begin{array}{l}\text { UK } \\
n=929\end{array}$ & p Value $\left(\chi^{2)}\right.$ & $\begin{array}{l}\text { Combined } \\
N=5106\end{array}$ \\
\hline Included after missing values removed & $477(99.0 \%)$ & $640(99.2 \%)$ & $867(96.7 \%)$ & $2037(94.6 \%)$ & $904(97.3 \%)$ & & $4925(96.5 \%)$ \\
\hline \multicolumn{8}{|l|}{ Sex } \\
\hline Female & $180(37.3)$ & $266(41.6 \%)$ & $411(47.4 \%)$ & $844(41.4 \%)$ & $352(38.9 \%)$ & & $2053(41.7 \%)$ \\
\hline Male & $297(62.3)$ & $374(58.4 \%)$ & $456(52.6 \%)$ & $1193(58.6 \%)$ & $552(61.1 \%)$ & $<0.001$ & $2872(58.3 \%)$ \\
\hline \multicolumn{8}{|l|}{ Age of onset } \\
\hline Median & 61.4 & 63.0 & 66.8 & 63.0 & 61.3 & & 63.1 \\
\hline \multicolumn{8}{|l|}{ Diagnostic delay } \\
\hline Short & $161(33.8 \%)$ & $193(30.2 \%)$ & $285(32.9 \%)$ & $702(43.5 \%)$ & $300(33.2 \%)$ & & $1641(33.3 \%)$ \\
\hline Medium & $163(34.2 \%)$ & $211(33.0 \%)$ & $293(33.8 \%)$ & $683(33.5 \%)$ & $298(33.0 \%)$ & & $1648(33.5 \%)$ \\
\hline Long & $153(32.1 \%)$ & $236(36.9 \%)$ & $289(33.3 \%)$ & $652(32 \%)$ & $306(33.8 \%)$ & 0.559 & $1636(33.2 \%)$ \\
\hline \multicolumn{8}{|l|}{ Site of onset } \\
\hline Spinal & $326(68.3 \%)$ & $437(68.3 \%)$ & $583(67.2 \%)$ & $1333(65.4 \%)$ & $610(67.5 \%)$ & & $3289(66.8 \%)$ \\
\hline Bulbar & $151(31.7 \%)$ & $203(31.7 \%)$ & $284(32.8 \%)$ & 704 (34.6\%) & $294(32.5 \%)$ & 0.540 & $1636(33.2 \%)$ \\
\hline \multicolumn{8}{|l|}{ C9orf72 } \\
\hline Normal & $392(82.2 \%)$ & $578(90.3 \%)$ & 805 (92.8\%) & 1861 (91.4\%) & $841(93.0 \%)$ & & 4477 (90.9\%) \\
\hline Expanded & $85(17.8 \%)$ & $62(9.7 \%)$ & $62(7.2 \%)$ & $176(8.6 \%)$ & $63(7 \%)$ & $<0.001$ & 448 (9.1\%) \\
\hline
\end{tabular}




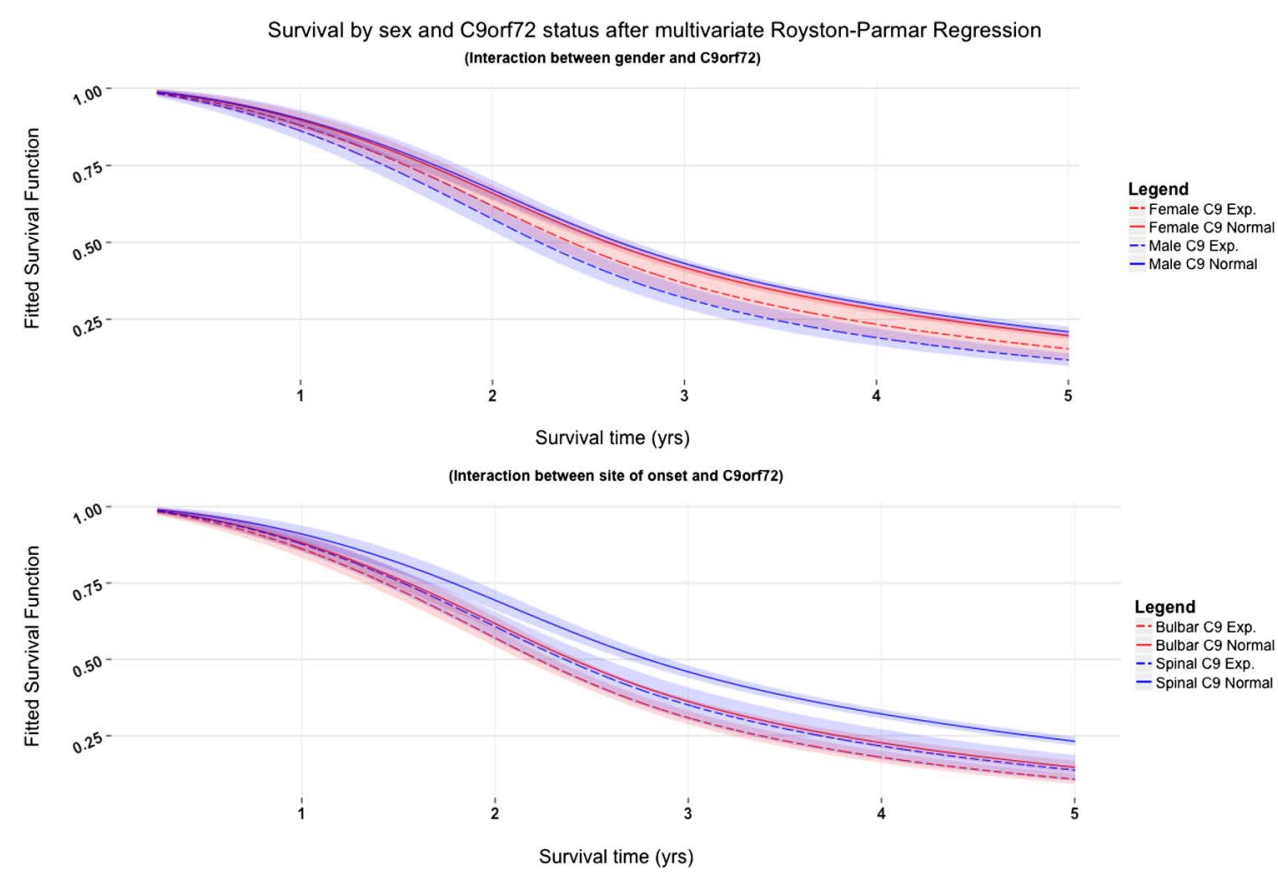

Figure 1 Predicted survival by sex and C9orf72 status after multivariate Royston-Parmar regression including interaction terms. C9 normal=group not carrying the C9orf72 expansion; C9 Exp=group carrying the C9orf72 expansion present. Shaded areas represent $95 \%$ Cls. Predicted survival curves for interaction models between gender and C9orf72 status (upper), and site of onset and C9orf72 status (lower) after multivariate regression using a Royston-Parmar model on the hazard scale (three degrees of freedom), correcting for age of onset (time varying), diagnostic delay group (time varying), site of onset, country and using a variance-covariance matrix clustered by country. The upper graph showing a wider spread by C9orf72 status in males compared to females, while the lower graph showing a wider spread in spinal onset cases versus bulbar onset cases; however, HRs for these interactions were not significant.

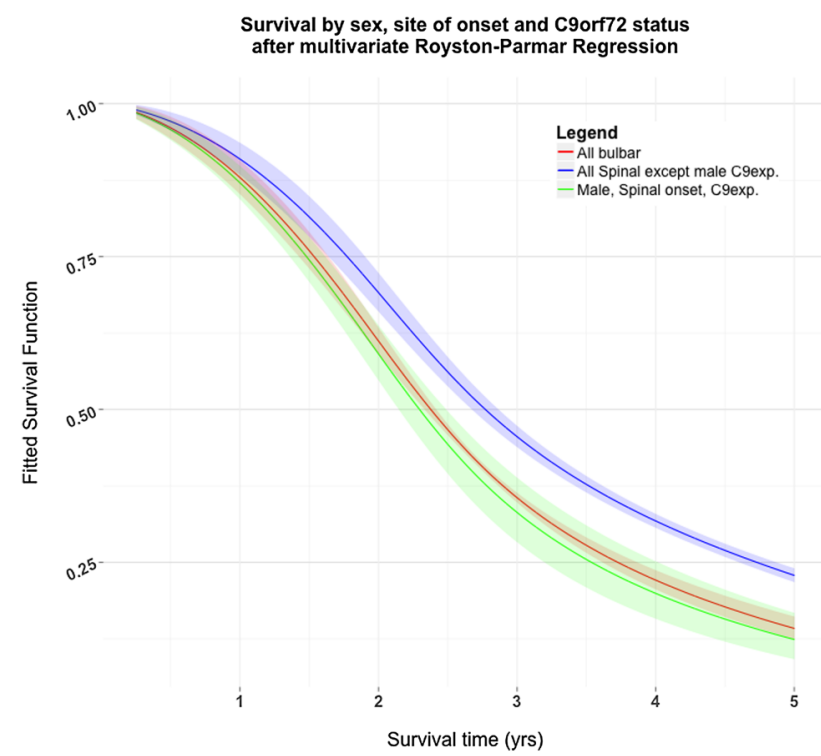

Figure 2 Predicted survival function for male spinal onset C9orf72 expanded patients. $C 9$ exp=group carrying the C9orf72 expansion. Shaded areas represent $95 \% \mathrm{Cls}$. Survival curves show three subgroups of patients categorised by sex, site of onset and C9orf72 status. Survival curves for male spinal onset patients with the C9orf72 repeat expansion are markedly worse than other spinal onset patients and are in fact inseparable from bulbar onset patients. Median predicted survival in the three groups were: spinal excluding C9orf72 expanded males $=2.77$ years $(95 \% \mathrm{Cl} 2.67$ to 2.87$)$; bulbar onset $=2.38$ years (95\% Cl 2.33 to 2.42); spinal onset males with C9orf72 repeat expansion $=2.29$ years $(95 \% \mathrm{Cl} 2.15$ to 2.49$)$. limitations in the power of previous studies due to lower numbers (table 4). Taken together, our findings and those of previous studies imply that distinct processes may operate in differing subgroups of ALS even when a known genetic factor is present as the underlying cause, and demonstrate that male gender is likely to be an important interacting factor in the biology of C9orf72-related disease.

A number of pathogenic mechanisms have been proposed to explain the role of the C9orf72 repeat expansion in ALS. These include haploinsufficiency, toxic RNA interfering with the function of RNA-binding proteins or other cellular factors, and the presence of toxic dipeptide repeat proteins through Repeatassociated non-ATG (RAN) translation. ${ }^{25} 26$ Recent work has also pointed towards C9orf72-induced pathology of nucleocytoplasmic transport processes. ${ }^{26-29}$ However, the pathobiology of the observed interaction between the C9orf72 variant and gender remains unclear, but it is congruent with observations in the SOD1 mouse model, in which transgenic mutant males have shorter survival compared to their transgenic female littermates with similar copy numbers. ${ }^{30}$ The mechanism for this gender effect in animals, although well recognised, has not been characterised, but can be attenuated when mice are bred on a different genetic background. ${ }^{30}$

A potential weakness of our study is that it did not include clinical scores for the presence of cognitive change, which is a known prognostic indicator in ALS. We and others have shown that those with $\mathrm{C} 9$ orf72 repeat expansions are more likely to experience cognitive and behavioural change; however, to the best of our knowledge, until now no gender-mediated effect has been demonstrated in C9orf72-related cognitive profiling. Moreover, since C9orf72 is part of the causal pathway for some 


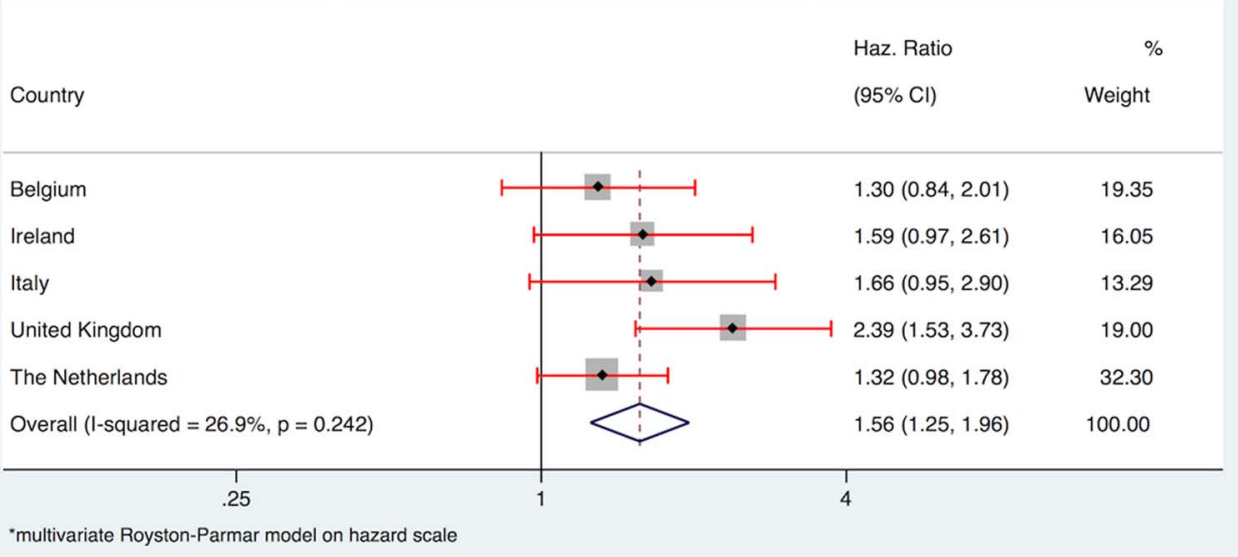

Figure 3 Individual patient data meta-analysis of the HR of male spinal onset patients with amyotrophic lateral sclerosis (ALS) carrying the C9orf72 repeat expansion. C9orf72exp, group carrying the C9orf72 expansion. IPD meta-analysis of C9orf72 repeat expansion in male spinal onset patients with ALS versus spinal onset C9orf72 normal patients pooled by country and analysed using a Royston-Parmar flexible parametric model with three degrees of freedom on the hazard scale correcting for age at onset (time varying), site of onset and diagnostic delay, based on the three level categorical breakdown of sex, site and C9orf72 status described in Supplement information 2. IPD, individual patient data.

Table 2 Age of onset and diagnostic delay for hybrid sex/site of onset/C9 variable

\begin{tabular}{|c|c|c|c|c|}
\hline \multirow[b]{2}{*}{ Level } & \multirow{2}{*}{$\begin{array}{l}\text { Age at onset } \\
\text { Median (IQR) }\end{array}$} & \multicolumn{3}{|c|}{ Diagnostic delay } \\
\hline & & Short & Medium & Long \\
\hline Spinal onset excluding C9orf72 expanded males & $62.3(54.0$ to 69.7$)$ & $965(30.8 \%)$ & $1014(32.4 \%)$ & $1151(36.8 \%)$ \\
\hline All bulbar onset & $65.0(58.4$ to 71.9$)$ & $611(37.4 \%)$ & $590(36.1 \%)$ & $435(26.6 \%)$ \\
\hline Male spinal onset C9orf72 only & $59.3(52.3$ to 64.7$)$ & $65(40.8 \%)$ & $44(27.7 \%)$ & $50(31.5 \%)$ \\
\hline$p$ Value & $0.0001^{*}$ & $<0.001 \dagger$ & & \\
\hline
\end{tabular}

${ }^{*}$ Kruskal-Wallis test.

$+\chi^{2}$ test.

Table 3 HRs for El-Escorial criteria after inclusion in final model

\begin{tabular}{|c|c|c|c|}
\hline El-Escorial category & HR & $95 \% \mathrm{Cl}$ & Wald test \\
\hline Suspected & 0.86 & 0.68 to 1.08 & 0.199 \\
\hline Possible & 1 & - & - \\
\hline Probable-laboratory supported & 1.38 & 1.31 to 1.44 & $<0.001$ \\
\hline Probable & 1.49 & 1.39 to 1.60 & $<0.001$ \\
\hline Definite & 2.09 & 1.99 to 2.21 & $<0.001$ \\
\hline \multicolumn{4}{|l|}{ Sex/site of onset/C9orf72 } \\
\hline Spinal onset excluding male C9orf72 expanded cases & 1 & - & - \\
\hline All bulbar onset cases & 1.33 & 1.21 to 1.45 & $<0.001$ \\
\hline Male C9orf72 expanded cases only & 1.57 & 1.26 to 1.96 & $<0.001$ \\
\hline
\end{tabular}

forms of FTD, inclusion of cognitive status as a variable would have introduced a selection bias based on 'conditioning on a common effect'. ${ }^{31}$ A further limitation to this study is that our analysis does not include C9orf72 repeat expansion analysis by Southern blot, although individual definition of pathological expansion performed by each centre using repeat primed PCR was validated by Southern blot. While the length of expansion varies from tissue to tissue, ${ }^{32-34}$ diagnostic testing within a clinical setting uses blood samples from which all previous prognostic and clinical correlative studies have been performed. Finally, we did not account for riluzole use in the analysis, as this is routinely prescribed in all patients from the participating countries, and it was not possible to determine the level of compliance using available data.

In conclusion, we have performed an analysis of the effect of the C9orf72 expansion on survival in almost 5000 European 
Table 4 Summary of previous analyses of survival by C9orf72 status

\begin{tabular}{|c|c|c|c|c|c|c|}
\hline Study & Population & $\begin{array}{l}\text { C9orf72 } \\
\text { normal }\end{array}$ & $\begin{array}{l}\text { C9orf72 } \\
\text { expanded }\end{array}$ & $\begin{array}{l}\text { Median survival } \delta^{*} \\
\text { (months) }\end{array}$ & $\begin{array}{l}\text { Median age at onset } \delta^{*} \\
\text { (years) }\end{array}$ & HR $95 \%(\mathrm{Cl})$ \\
\hline Byrne et $a l^{4}$ & Ireland & 170 & 21 & -6 & -3.2 & 1.9 (1.1 to 3.7$)$ \\
\hline Van Rheenen et $a P^{5}$ & The Netherlands & 1422 & 78 & -2.5 & -2.6 & $1.46(1.17$ to 1.83$)$ \\
\hline Sabatelli et $a l^{6}$ & Italy and Sardinia & 1688 & 69 & -12 & -3.8 & 1.79 (1.26 to 2.98$)$ \\
\hline Borghero et $\mathrm{al}^{7}$ & Sardinia & 375 & 51 & $-18 t$ & -0.9 & NA \\
\hline Debray et $a l^{8}$ & Belgium & 513 & 77 & $\begin{array}{l}\text { fALS }-38.3 \\
\text { sALS }-5.8\end{array}$ & $\begin{array}{l}\text { fALS }-5.9 \\
\text { sALS }-0.3\end{array}$ & $\begin{array}{l}\text { fALS } 2.5 \text { (1.5 to } 4.3) \\
\text { sALS } 1.1 \text { (0.8 to } 1.5)\end{array}$ \\
\hline $\begin{array}{l}\text { García-Redondo } \\
\text { et al }{ }^{9}\end{array}$ & Spanish & 936 & 67 & -12 & $-2.6 \ddagger$ & NA \\
\hline Irwin et $\left.a\right|^{10} \S$ & $\begin{array}{l}\text { USA } \\
\text { (Pennsylvania) }\end{array}$ & 69 & 64 & $-6 \ddagger$ & $-3.0 \ddagger$ & NA \\
\hline
\end{tabular}

*Negative figures imply C9orf72 expanded survive for shorter time, or are younger at onset than C9orf72 normal cases.

tCalculate as median survival in C9orf72 expanded group-median survival in overall cohort median.

$¥$ Calculated from mean data instead of median.

§Mixed ALS and FTD cases.

fALS, familial amyotrophic lateral sclerosis; sALS, sporadic amyotrophic lateral sclerosis.

patients with ALS. We have shown, for the first time, that C9orf72 repeat expansion is a significant negative prognostic indicator in males with spinal onset disease only. These findings suggest a hitherto unrecognised interaction between the C9orf repeat expansion, site of onset and gender. This has important implications in the understanding both the pathobiology of C9orf72-mediated disease, and in the development of future disease-related prognostic models.

\section{Author affiliations}

${ }^{1}$ Academic Unit of Neurology, Trinity Biomedical Sciences Institute, Trinity College, Dublin, Ireland

${ }^{2}$ Department of Basic and Clinical Neuroscience, Maurice Wohl Clinical Neuroscience Institute, Institute of Psychiatry, Psychology and Neuroscience, King's College London, London, UK

${ }^{3}$ Department of Neurology and Neurosurgery, Brain Center Rudolf Magnus, University Medical Center Utrecht, Utrecht, The Netherlands

${ }^{4}$ Department of Neuroscience, ALS Centre, University of Torino, Turin, Italy ${ }^{5}$ Department of Neurology, ALS Center, Azienda Ospedaliera Universitaria Maggiore Della Carità, Novara, Italy

${ }^{6}$ Faculty of Medicine, University of Southampton, Southampton, UK

${ }^{7}$ Sheffield Institute for Translational Neuroscience, University of Sheffield, Sheffield, UK

${ }^{8}$ Department of Neurosciences, Experimental Neurology—Laboratory of Neurobiology, KU Leuven—University of Leuven, Belgium

${ }^{9}$ Vlaams Instituut voor Biotechnologie (VIB), Vesalius Research Center, Laboratory of Neurobiology, Leuven, Belgium

${ }^{10}$ Department of Neurology, University Hospital Leuven, Leuven, Belgium

${ }^{11}$ Department of Neurology, Beaumont Hospital, Dublin, Ireland

Acknowledgements The authors would like to thank people with MND and their families for their participation in this project. They also acknowledge sample management undertaken by Biobanking Solutions funded by the Medical Research Council at the Centre for Integrated Genomic Medical Research, University of Manchester.

Contributors JR had full access to all the data in the study and takes responsibility for the integrity of the data and the accuracy of the data analysis. JR, IF and $\mathrm{OH}$ were involved in study concept and design. H-JW, AV, RML, MH, AJ, RvE, AC, LM, CS, KM, PJS, WR, PVD, AA-C; LvdB; AC; JV and $\mathrm{OH}$ were involved in acquisition of data. JR; $\mathrm{H}$-JW and JV contributed to statistical analysis. JR and $\mathrm{OH}$ contributed to drafting of the manuscript. All authors were involved in critical revision of the manuscript for important intellectual content.

Funding This work was supported by the Interuniversity Attraction Poles (IUAP) programme P7/16 of the Belgian Federal Science Policy Office, by the FWOVlaanderen, and ZonMw under the frame of E-Rare-2, the ERA-Net for Research on Rare Diseases (PYRAMID). The research leading to these results has received funding from the Health Research Board Interdisciplinary Capacity Enhancement Programme, the European Community's Seventh Framework Programme (FP7/2007-2013) under the Health Cooperation Programme and the project EUROMOTOR (number 259867)
This work was funded in part by Fondazione Vialli e Mauro per la Sclerosi Laterale Amiotrofica onlus, the Italian Ministry of Health (Ricerca Sanitaria Finalizzata, 2010, grant RF-2010-2309849). This project was supported by the charity Research Motor Neurone, and the Irish Health Research Board, SOPHIA and STRENGTH which are European Joint Programme - Neurodegenerative Disease Research (JPND) projects. The projects are supported through the following funding organisations under the aegis of JPND-http://www. jpnd.eu (UK, Medical Research Council and Economic and Social Research Council; Netherlands, ZonMW; Italy, MUIR; Belgium FWOVlaanderen) and the Health Research Board of Ireland. JR was funded by the Health Research Programme Clinical Fellowship Programme (grant number HPF-2014-527). IF was supported by funds from Motor Neurone Disease Association of Great Britain and Northern Ireland (grant number 905-793, 6058). PVD holds a clinical investigatorship from FWO-Vlaanderen and is supported by the ALS liga Belgium. WR is supported by the European Community's Health Seventh Framework Programme (FP7/2007-2013) under grant numbers 259867 \& 340429, by funding from Opening the Future Fund, Hart voorALS fund and by the E von Behring Chair for Neuromuscular and NeurodegenerativeDisorders, all at the University of Leuven, and by funding from the ALS LeagueBelgium, the Thierry Latran Foundation and the Geneeskundige Stichting KoninginElisabeth (G.S.K.E.). PJS is supported as an NIHR Senior Investigator. CES and AAC receive salary support from the National Institute for Health Research (NIHR) Dementia Biomedical Research Unit at South London and Maudsley NHS Foundation Trust and King's College London. PJS is supported as an NIHR Senior Investigator. LvB received a grant from the Netherlands Organization for Health Research and Development (Vici scheme).

Samples used in this research were in part obtained from the UK National DNA Bank for MND Research, funded by the MND Association and the Wellcome Trust.

Disclaimer The funders played no role in the design and conduct of the study; collection, management, analysis, and interpretation of the data and preparation, review or approval of the manuscript.

Competing interests $\mathrm{AA}-\mathrm{C}$ is chief investigator of a clinical trial by OrionPharma, served on the scientific advisory board of the Motor Neurone Disease Association, is on the Editorial Board of the journals 'F1000' and 'Amyotrophic Lateral Sclerosis and Frontotemporal Dementia', has consulted for GSK, OrionPharma, Biogen Inc, Cytokinetics, and Treeway, and receives royalties from the books 'The Brain' (Oneworld Publications) and 'The Genetics of Complex Human Diseases" (Cold Spring Harbor Laboratory Press). PJS is Principal Investigator of a clinical trial by OrionPharma, served on the scientific advisory board of the UK Medical Research Council, is on the Editorial Board of the 'Amyotrophic Lateral Sclerosis and Frontotemporal Dementia Journal', has consulted for, OrionPharma, Biogen, Cytokinetics, Treeway and Sanofi Aventis and receives royalties from the books 'Oxford Textbook of Neurology. She has grants from the Motor Neurone Disease Association, National Institute for Health Research, European Commission, Medical Research Council and ALS Worldwide. AC serves on the editorial advisory board of the journal 'Amyotrophic Lateral Sclerosis and Frontotemporal Dementia' and has been a member of advisory panels Biogen Idec, Cytokinetics, Italfarmaco and Neuraltus. $\mathrm{OH}$ is funded by the Health Research Board Clinician Scientist Programme. Professor Hardiman has received speaking honoraria from Novarits, Biogen Idec, Sanofi Aventis and Merck-Serono. She has been a member of advisory panels for Biogen Idec, Allergen, Ono pharmaceuticals, Novartis, Cytokinetics and Sanofi Aventis. She serves as Editor-in-Chief of the journal 'Amyotrophic Lateral 
Sclerosis and Frontotemporal Dementia'. LvB received travel grants and consultancy fees from Baxalta; serves on scientific advisory boards for Prinses Beatrix Spierfonds, Thierry Latran Foundation, Cytokinetics and Biogen. He serves on the editorial boards of 'Amyotrophic Lateral Sclerosis and Frontotemporal Dementia' and 'Journal of Neurology, Neurosurgery and Psychiatry'.

Patient consent Obtained.

Ethics approval Ethical approval for this study and data sharing was obtained at each participating centre, and data shared through a legal agreement under the auspices of the EU JPND STRENGTH consortium, administered through King's College London. For the purposes of this analysis, overarching approval was through the Irish Centre (Trinity College Dublin and Beaumont Hospital Research Ethics Committee (02/28; 05/49).

Provenance and peer review Not commissioned; externally peer reviewed.

\section{REFERENCES}

1 Al-Chalabi A, Hardiman O. The epidemiology of ALS: a conspiracy of genes, environment and time. Nat Rev Neurol 2013;9:617-28.

2 DeJesus-Hernandez M, Mackenzie IR, Boeve BF, et al. Expanded GGGGCC hexanucleotide repeat in noncoding region of C9ORF72 causes chromosome 9p-linked FTD and ALS. Neuron 2011;72:245-56.

3 Renton AE, Majounie E, Waite $A$, et al. A hexanucleotide repeat expansion in C9ORF72 is the cause of chromosome 9p21-linked ALS-FTD. Neuron 2011:72:257-68.

4 Byrne S, Elamin M, Bede P, et al. Cognitive and clinical characteristics of patients with amyotrophic lateral sclerosis carrying a C9orf72 repeat expansion: a population-based cohort study. Lancet Neurol 2012;11:232-40.

5 van Rheenen W, van Blitterswijk M, Huisman MHB, et al. Hexanucleotide repeat expansions in C9ORF72 in the spectrum of motor neuron diseases. Neurology 2012:79:878-82.

6 Sabatelli M, Conforti FL, Zollino M, et al. C9ORF72 hexanucleotide repeat expansions in the Italian sporadic ALS population. Neurobiol Aging 2012;33:1848. e15-20.

7 Borghero G, Pugliatti M, Marrosu F, et al. Genetic architecture of ALS in Sardinia. Neurobiol Aging 2014;35:2882.e7-e12.

8 Debray S, Race V, Crabbé V, et al. Frequency of C9orf72 repeat expansions in amyotrophic lateral sclerosis: a Belgian cohort study. Neurobiol Aging 2013;34:2890.e7-e12.

9 García-Redondo A, Dols-Icardo 0, Rojas-García R, et al. Analysis of the C9orf72 Gene in patients with amyotrophic lateral sclerosis in Spain and different populations worldwide. Hum Mutat 2013;34:79-82.

10 Irwin DJ, McMillan CT, Brettschneider J, et al. Cognitive decline and reduced survival in C9orf72 expansion frontotemporal degeneration and amyotrophic lateral sclerosis. J Neurol Neurosurg Psychiatr 2013;84:163-9.

11 ENCALS. ALS Core Clinical Dataset. 2014. http://www.encals.eu/public/media/ ac8bacd302965623601ddfe4229c4aadcc370922/6xg7/pdf/

17a00a103f602f2837d968fd038df3ca17ae0c76.pdf

12 Byrne S, Heverin M, Elamin M, et al. Aggregation of neurologic and neuropsychiatric disease in amyotrophic lateral sclerosis kindreds: a population-based case-control cohort study of familial and sporadic amyotrophic lateral sclerosis. Ann Neurol 2013;74:699-708.

13 Rooney J, Vajda A, Heverin M, et al. Spatial cluster analysis of population amyotrophic lateral sclerosis risk in Ireland. Neurology 2015;84:1537-44.
14 Huisman MHB, de Jong SW, van Doormaal PTC, et al. Population based epidemiology of amyotrophic lateral sclerosis using capture-recapture methodology. J Neurol Neurosurg Psychiatr 2011;82:1165-70.

15 Migliaretti G, Berchialla P, Dalmasso P, et al. Amyotrophic lateral sclerosis in Piedmont (Italy): a Bayesian spatial analysis of the incident cases. Amyotroph Lateral Scler Frontotemporal Degener 2013;14:58-65.

16 Beghi E, Logroscino G, Chiò A, et al. The epidemiology of ALS and the role of population-based registries. Biochim Biophys Acta 2006;1762:1150-7.

17 Pupillo E, Messina P, Giussani G, et al. Physical activity and amyotrophic lateral sclerosis: a European population-based case-control study. Ann Neurol 2014;75:708-16.

18 van Rheenen W, Diekstra FP, van Doormaal PTC, et al. H63D polymorphism in HFE is not associated with amyotrophic lateral sclerosis. Neurobiol Aging 2013;34:1517. e5-7.

19 van Es MA, Veldink JH, Saris CGJ, et al. Genome-wide association study identifies 19p13.3 (UNC13A) and 9p21.2 as susceptibility loci for sporadic amyotrophic lateral sclerosis. Nat Genet 2009:41:1083-7.

20 Royston P, Lambert PC. Flexible Parametric Survival Analysis Using Stata: Beyond the Cox Model, First Edit. Stata Press, 2011. http://www.stata.com/bookstore/ flexible-parametric-survival-analysis-stata/

21 Lambert P. STPM2: Stata module to estimate flexible parametric survival models. 2012. http://ideas.repec.org/c/boc/bocode/s457128.html

22 Fisher DJ. Two-stage individual participant data meta-analysis and generalized forest plots. Stata Journal 2015;15:369-96.

23 Wickham H. Ggplot2: Elegant Graphics for Data Analysis. New York: Springer-Verlag, 2009. http://had.co.nz/ggplot2/book

24 Watanabe $\mathrm{H}$, Atsuta N, Nakamura R, et al. Factors affecting longitudinal functional decline and survival in amyotrophic lateral sclerosis patients. Amyotroph Lateral Scler Front Degener 2015;16:230-6.

25 Bennett W. Fox RST. Problems at the nuclear pore expansion. Nature 2015;525: 36-7.

26 Xiao S, MacNair L, McGoldrick P, et al. Isoform-specific antibodies reveal distinct subcellular localizations of C9orf72 in amyotrophic lateral sclerosis. Ann Neurol 2015;78:568-83

27 Jovičić A, Mertens J, Boeynaems S, et al. Modifiers of C9orf72 dipeptide repeat toxicity connect nucleocytoplasmic transport impairments to FTD/ALS. Nat Neurosci 2015;18:1226-9.

28 Freibaum BD, Lu Y, Lopez-Gonzalez R, et al. GGGGCC repeat expansion in C9orf72 compromises nucleocytoplasmic transport. Nature 2015:525:129-33.

29 Zhang K, Donnelly CJ, Haeusler AR, et al. The C9orf72 repeat expansion disrupts nucleocytoplasmic transport. Nature 2015;525:56-61.

30 Heiman-Patterson TD, Deitch JS, Blankenhorn EP, et al. Background and gender effects on survival in the TgN(SOD1-G93A)1Gur mouse model of ALS. J Neurol SCi 2005:236:1-7.

31 Cole SR, Platt RW, Schisterman EF, et al. Illustrating bias due to conditioning on a collider. Int J Epidemiol 2010;39:417-20.

32 van Blitterswijk $\mathrm{M}$, DeJesus-Hernandez $\mathrm{M}$, Niemantsverdriet $\mathrm{E}$, et al. Association between repeat sizes and clinical and pathological characteristics in carriers of C9ORF72 repeat expansions (Xpansize-72): a cross-sectional cohort study. Lancet Neurol 2013:12:978-88.

33 Cooper-Knock J, Shaw PJ, Kirby J. The widening spectrum of C9ORF72-related disease; genotype/phenotype correlations and potential modifiers of clinical phenotype. Acta Neuropathol 2014;127:333-45.

34 Gendron TF, van Blitterswijk M, Bieniek KF, et al. Cerebellar c9RAN proteins associate with clinical and neuropathological characteristics of C9ORF72 repeat expansion carriers. Acta Neuropathol 2015;130:559-73. 